\title{
Downregulation of the neuronal opioid gene expression concomitantly with neuronal decline in dorsolateral prefrontal cortex of human alcoholics
}

Igor Bazov $\mathbb{D}^{1}$, Daniil Sarkisyan', Olga Kononenko', Hiroyuki Watanabe', Victor M. Karpyak², Tatiana Yakovleva ${ }^{1}$ and Georgy Bakalkin ${ }^{1}$

\begin{abstract}
Molecular changes in cortical areas of addicted brain may underlie cognitive impairment and loss of control over intake of addictive substances and alcohol. Prodynorphin (PDYN) gives rise to dynorphin (DYNs) opioid peptides which target kappa-opioid receptor (KOR). DYNs mediate alcohol-induced impairment of learning and memory, while KOR antagonists block excessive, compulsive-like drug and alcohol self-administration in animal models. In human brain, the DYN/KOR system may undergo adaptive changes, which along with neuronal loss, may contribute to alcoholassociated cognitive deficit. We addressed this hypothesis by comparing the expression levels and co-expression (transcriptionally coordinated) patterns of PDYN and KOR (OPRK1) genes in dorsolateral prefrontal cortex (dIPFC) between human alcoholics and controls. Postmortem brain specimens of 53 alcoholics and 55 controls were analyzed. PDYN was found to be downregulated in dIPFC of alcoholics, while OPRK1 transcription was not altered. PDYN downregulation was confined to subgroup of subjects carrying C, a high-risk allele of PDYN promoter SNP rs1997794 associated with alcoholism. Changes in PDYN expression did not depend on the decline in neuronal proportion in alcoholics, and thereby may be attributed to transcriptional adaptations in alcoholic brain. Absolute expression levels of PDYN were lower compared to those of OPRK1, suggesting that PDYN expression is a limiting factor in the DYN/KOR signaling, and that the PDYN downregulation diminishes efficacy of DYN/KOR signaling in dIPFC of human alcoholics. The overall outcome of the DYN/KOR downregulation may be disinhibition of neurotransmission, which when overactivated could contribute to formation of alcohol-related behavior.
\end{abstract}

\section{Introduction}

Alcohol consumed in moderate and large amounts causes acute and delayed impairments in cognitive and executive functions that guide complex behavior through planning, decision-making, and response control ${ }^{1-6}$. Total alcohol consumption and binge drinking patterns are risk

\footnotetext{
Correspondence: Igor Bazov (bazov@yahoo.com) or Georgy Bakalkin (Georgy. Bakalkin@farmbio.uu.se)

'Division of Biological Research on Drug Dependence, Department of Pharmaceutical Biosciences, Uppsala University, SE-751 24 Uppsala, Sweden ${ }^{2}$ Department of Psychiatry and Psychology, Mayo Clinic College of Medicine, Rochester, MN 55905, USA
}

factors for dementia ${ }^{7-9}$. Alcohol-induced cognitive deficit is a factor underlying the habitual drug seeking and taking that characterize addiction and dependence ${ }^{10,11}$.

The mechanism of alcohol-induced cognitive impairments remains unknown but may involve neurodegeneration and aberrant neurotransmission. Several studies indicate that cognitive effects of alcohol may be mediated through dysregulation of the dynorphin/K-opioid receptor (DYN/KOR) system in the prefrontal cortex (PFC) and hippocampus ${ }^{12-16}$. DYN opioid peptides have been implicated in cognitive decline ${ }^{17-22}$. Administration of synthetic DYN into dorsal hippocampus impairs spatial 
learning in rats ${ }^{17}$. DYNs contribute to age-related and stress-induced deficits in learning and memory ${ }^{18-20}$. In elderly humans, prodynorphin $(P D Y N)$ gene polymorphisms play a role in memory function ${ }^{22}$. In individuals with Alzheimer's disease, DYN is elevated in PFC and this increase correlates with neuropathological lesions ${ }^{21}$.

A role of the DYN/KOR system in impairment of spatial learning and memory was identified in a rat model of cognitive deficit induced by alcohol binge drinking ${ }^{16,23,24}$. Selective KOR antagonist nor-binaltorphimine normalized animal performance in spatial learning and memory tasks possibly by reversion of ethanol-induced elevation in glutamate overflow.

Molecular and cellular allostatic changes in addicted brain may underlie addictive behavior and associated phenomena including impairment of cognitive functions ${ }^{25}$. Changes in the DYN/KOR system may be involved in allostatic processes in the addicted brain as demonstrated in animal studies ${ }^{16,26-34}$. The aim of the present study was to examine whether the DYN/KOR system undergoes adaptive changes at the transcriptional level in dorsolateral prefrontal cortex (dlPFC) in human alcoholics. We previously conducted a pilot analysis of a limited number of human alcoholics and controls that revealed no differences in the expression of the $P D Y N$ gene in the orbitofrontal cortex (OFC) and OPRK1 gene (opioid receptor kappa 1 gene encoding KOR protein) in dIPFC, and elevation with a borderline significance of $P D Y N$ and KOR mRNA levels in $\mathrm{dIPFC}$ and OFC, respectively, of alcoholics ${ }^{35}$. The number of subjects is a critical factor in molecular analysis of human brain. Reasoning that our previous study was possibly underpowered, in the present work the number of subjects was increased nearly 4-fold and more stringent statistical analysis was applied.

Analysis of absolute expression levels revealed a coregulated (transcriptionally coordinated) pattern of PDYN (prodynorphin gene encoding precursor of DYN peptides) and OPRK1 expression in human nucleus accumbens $(\mathrm{NAc})^{36}$. This pattern was significantly different between alcoholics and controls. To assess whether the PDYN and OPRK1 genes are co-regulated in dIPFC, and whether this co-regulation is altered in addicted dIPFC, we compared $P D Y N$ and OPRK1 co-expression along with average expression levels of these genes in dlPFC between alcoholics and controls.

A number of neurons is markedly reduced in alcoholics in dIPFC as demonstrated in early postmortem morphological studies ${ }^{37,38}$ and confirmed by analyses of neuronal proportion quantified from epigenome-wide DNA methylation profiles and expression of neuronal marker (RBFOX3 encoding NeuN protein) ${ }^{39}$. To attribute potential expression changes to transcriptional events or changes in cell composition in alcoholics, we examined whether PDYN and KOR (OPRK1) mRNAs are expressed strictly in neurons in human brain and then analyzed effects of the decline in neuronal proportion and RBFOX3 expression on $P D Y N$ and OPRK1 mRNA alterations in alcoholic brain. Effects of PDYN and OPRK1 singlenucleotide polymorphisms (SNPs) strongly associated with alcoholism on expression of both genes were also studied.

\section{Materials and methods Human samples}

Human frozen brain tissues were collected at the New South Wales Brain Tissue Resource Centre (NSW BTRC), University of Sydney, Australia (https://sydney.edu.au/ medicine/pathology/btrc/; see Table 1 for short summary and Supplementary Table 1 for detailed information). Tissue samples from 55 control and 53 alcoholic subjects, all males of European descent, were analyzed. Alcoholics were the subjects that met Diagnostic and Statistical Manual for Mental Disorders, 4th edition (DSM-IV) criteria for Alcohol Abuse or Alcohol Dependence and consumed $206 \pm 20 \mathrm{~g}$ (mean \pm S.E.M.) of ethanol per day in average for the majority of their adult lives ${ }^{40}$. Controls had either abstained from alcohol completely or were social drinkers who consumed $17 \pm 3 \mathrm{~g}$ of ethanol per day on average. Methods used to classify alcoholics were described previously ${ }^{40,41}$. Cases with a history of polydrug abuse (with evidence that the individual abused other drugs such as cocaine or heroin) or with medical complications such as Wernicke-Korsakoff syndrome or alcoholic cases with concomitant diseases were excluded. Cases with a history of cerebral infarction, head injury, or neurodegenerative disease (e.g., Alzheimer's disease) were also excluded. dlPFC samples were dissected from superior frontal gyrus/Brodmann area 9. Informed written consent for autopsy was obtained from the next-of-kin and collection was approved by the Human Research Ethics Committees of the Sydney Local Health District (X15-0199) and the University of Sydney. The study was approved by the Swedish Central Ethical Review Board. Smoking status information was available for $94 \%$ of subjects (Supplementary Table 1; "ex-smokers" were grouped with "non-smokers").

\section{RNA purification}

Total RNA was purified using RNeasy Lipid Tissue Mini Kit (Qiagen) and treated with RNase-free DNase I (Qiagen) on column, according to the manufacturer's recommendations. RNA concentrations and 260/280 and 260/230 ratios were measured with a Nanodrop. RNA quality indicator (RQI) was measured using Bio-Rad Experion (Bio-Rad) with Eukaryote Total RNA StdSens assay, according to the manufacturer's protocol. $500 \mathrm{ng}$ RNA were reverse-transcribed to cDNA in duplicates with the High-Capacity RNA-to-cDNA kit (Applied Biosystems), according to the manufacturer's recommendations. 
Table 1 Summary of demographic data and tissue characteristics of human subjects (for details, see Supplementary Table 1)

\begin{tabular}{|c|c|c|c|c|c|c|}
\hline & $N$ & Age & PMI & $\mathrm{pH}$ & RQI & Neuronal proportion ${ }^{\mathrm{a}}$ \\
\hline \multicolumn{7}{|c|}{ Analysis of gene expression } \\
\hline Controls & 55 & $54.7 \pm 9.1$ & $30.0 \pm 12.5$ & $6.6 \pm 0.2$ & $8.1 \pm 1.0$ & \\
\hline Alcoholics & 53 & $56.2 \pm 8.9$ & $35.8 \pm 15.3$ & $6.5 \pm 0.3$ & $7.4 \pm 1.3$ & \\
\hline$P$-value & & n.s. & 0.033 & 0.015 & 0.002 & \\
\hline \multicolumn{7}{|c|}{ Analysis of neuronal proportion } \\
\hline Controls & 35 & $54.9 \pm 9.6$ & $28.5 \pm 13.6$ & $6.6 \pm 0.2$ & $8.1 \pm 1.1$ & $0.24 \pm 0.04$ \\
\hline Alcoholics & 30 & $58.6 \pm 9.5$ & $31.9 \pm 15.3$ & $6.5 \pm 0.3$ & $7.4 \pm 1.3$ & $0.21 \pm 0.07$ \\
\hline$P$-value & & n.s. & n.s. & n.s. & 0.017 & 0.033 \\
\hline
\end{tabular}

\section{Gene expression analysis}

TaqMan assays (Applied Biosystems) for GFAP (Hs00909233_m1), OPRK1 (Hs00175127_m1), PDYN (Hs00225770_m1), POLR2A (Hs00172187_m1), RBFOX3 (Hs01370653_m1), and RPLPO (Hs99999902_m1) were used. cDNAs were mixed with TaqMan assay and iTaq Universal Probes supermix (Applied Biosystems) for qPCR with a CFX96 Real-Time Detection System (BioRad), according to the manufacturer's instructions. Levels of each gene of interest mRNA were normalized to geometric mean of expression levels of two control genes POLR2A and RPLPO selected by geNORM program (https://genorm.cmgg.be/) ${ }^{42}$ (see also our studies ${ }^{35,43,44}$ ). In each experiment, internal control gene-stability measure $M^{42}$ was controlled for and did not exceed the limit of 0.5 .

\section{Radioimmunoassay (RIA)}

The procedure has been described elsewhere ${ }^{45,46}$. Briefly, $1 \mathrm{M}$ hot acetic acid was added to finely powdered frozen brain tissues, and samples were boiled for $5 \mathrm{~min}$, ultrasonicated and centrifuged. Tissue extracts were run through SP-Sephadex ion exchange C-25 column, and peptides were eluted and analyzed by RIA. Anti-Dyn B antiserum showed $100 \%$ molar cross-reactivity with Big DYN, which consists of Dyn A and Dyn B sequences, $0.8 \%$ molar cross-reactivity with Leu-morphine (29 amino acid C-terminally extended Dyn B), and $<0.1 \%$ molar crossreactivity with Dyn A (1-17), Dyn A (1-8), $\alpha$-neoendorphin, and Leu-enkephalin ${ }^{47}$.

\section{Isolation of cell nuclei}

Tissue samples were Dounce homogenized in the lysis buffer $\left(0.32 \mathrm{M}\right.$ sucrose; $5 \mathrm{mM} \mathrm{CaCl}_{2} ; 3 \mathrm{mM}$ magnesium acetate; $0.1 \mathrm{mM}$ EDTA; $10 \mathrm{mM}$ Tris- $\mathrm{HCl}, \mathrm{pH}$ 8.0; 0.1\%
Triton X-100; 1 mM DTT). Homogenized samples were gently suspended in sucrose solution (1.7 $\mathrm{M}$ sucrose; $3 \mathrm{mM}$ magnesium acetate; $1 \mathrm{mM}$ DTT; $10 \mathrm{mM}$ Tris- $\mathrm{HCl}$, $\mathrm{pH}$ 8.0), and layered onto a sucrose cushion. Ultracentrifugation was carried out at $30,000 \times g$ for $2.5 \mathrm{~h}$ at $4^{\circ}$ C (Beckman; L8-70 M; SW28 swing bucket rotor). After centrifugation, the supernatant was removed by aspiration. Nuclei pellets resuspended in PBS were filtered through a $40 \mu \mathrm{m}$ Nitex mesh to remove remaining clumps.

\section{Flow cytometry}

Neuronal nuclei were isolated by fluorescence-activated nuclei sorting (FANS) after labeling with neuron-specific monoclonal antibody against NeuN (MAB377, Millipore). NeuN antibodies conjugated with mouse IgG labeling reagent (Alexa 488, Molecular Probes) were incubated with nuclear suspension for $30 \mathrm{~min}$ in the dark and directly sorted in the RLT lysis buffer (Qiagen). FANS was performed using a FACSAria III cell sorter (BD BioSciences), nuclei were pelleted by centrifugation at $3000 \mathrm{x} g$ for $5 \mathrm{~min}$ at $4{ }^{\circ} \mathrm{C}$, and stored at $-80{ }^{\circ} \mathrm{C}$. To ensure sorting of single but not aggregated nuclei preparations were stained with Hoesch dye, and a gate was set to isolate singlets only that were readily discerned from doublets, triplets, and higherorder aggregates based on their fluorescence intensity. The purity of neuronal nuclei was confirmed by FANS analysis of the sorted preparations.

\section{Droplet digital PCR}

The assay was described elsewhere ${ }^{48}$. Total RNA from FANS-sorted nuclei was purified using RNeasy Plus Mini kit (Qiagen) and 15-60 ng RNA were reverse-transcribed with the High-Capacity RNA-to-cDNA kit (Applied Biosystems), according to the manufacturer's recommendations. cDNAs 
were mixed with TaqMan assay, ddPCR Supermix for Probes (Bio-Rad) and Droplet Generation Oil (Bio-Rad), partitioned into 14,000-17,000 droplets in QX200 Droplet Generator and used for PCR with T100 Thermal Cycler (Bio-Rad), according to the manufacturer's instructions. The fluorescence intensity of the droplets was measured using the QX200 Droplet Reader (Bio-Rad). The data analysis was performed with QuantaSoft droplet reader software (BioRad). mRNA amount was calculated using the Poisson statistics ${ }^{49}$. The absolute transcript levels were expressed in mRNA copies per ng of total RNA. Correlation between $P D Y N$ expression levels obtained using ddPCR and qRTPCR was positive and significant (Pearson $R=0.9, P=$ 0.0004) (Supplementary Fig. 1).

\section{Genotyping}

SNPs were determined by SNP\&SEQ Technology Platform at Uppsala University using Illumina HumanOmni5Exome-4v1 beadchip.

\section{Computation of neuronal proportions}

Genome-wide DNA methylation data for 482,421 CpGs in DNA from total tissue was profiled by SNP\&SEQ Technology Platform at Uppsala University using Illumina Infinium HumanMethylation 450 beadchip and processed using R package Cell EpigenoType-Specific CETS mapper. CETS predicts neuronal proportions from methylation levels of the top 10,000 marker CpGs, which demonstrated the most significant methylation differences between neuronal and non-neuronal DNA ${ }^{39,50}$.

\section{Statistical analysis}

Statistical analysis was performed using $\mathrm{R}$ version 3.3.2 (https://www.R-project.org/). Statistica 13 (StatSoft Scandinavia, Sweden) was used for analysis of absolute expression levels by unpaired Student's $t$-test and for sample size calculation of one-way ANOVA by statistical power analysis with target Type I error $\alpha=0.05$ and statistical power $\beta=0.8$. For analysis of gene expression linear regression model adjusting for age, PMI, brain $\mathrm{pH}$, RQI, and alcoholism followed by post hoc Tukey HSD test on least squares means was performed using car and lsmeans packages. Gene expression was adjusted for RBFOX3 mRNA levels as a surrogate measure of neuronal proportion and/or activity; it was further adjusted for neuronal proportion instead of RBFOX3 mRNA levels. An interaction effect between alcoholism and KOR (OPRK1) mRNA levels was also considered. Smoking, mean and total alcohol consumption, DSM-V severity of alcohol use disorder and four alcoholism-associated SNPs were added to the model one at a time to test if they can account for gene expression variance. Overly influential points with Cook's distance $\geq 1.0$ were removed from analysis of the above models ${ }^{51}$. One subject was excluded as a biological outlier; and two subjects were excluded as statistical outliers. $R$ package effects was used to construct effect displays (component and residual plots). Bootstrapped $P$ values, and bias-corrected and accelerated bootstrap percentile 95\% confidence intervals (CI) for regression coefficients, both of which do not require the assumption of normality, were estimated using car package with $R=$ $5 \times 10^{5}$ resampled cases ${ }^{52}$. Script to analyze data and generate figures is written using publicly available software $\mathrm{R}$ and is available from authors upon request. A significance level of $P<0.05$ was accepted as statistically significant and all tests were two-tailed.

\section{Results}

Fifty-three DSM-IV alcoholic and 55 control subjects were analyzed in the study. Effects of alcoholism on the whole tissue levels of PDYN and OPRK1 mRNAs in dIPFC were examined after adjusting for demographical data and tissue characteristics including age, PMI, brain $\mathrm{pH}$, and RQI (Table 1). We and others demonstrated that the number of neurons in dIPFC is markedly reduced in alcoholics $^{37-39}$. Tissue expression levels may differ between the subject groups due to changes in cell composition if genes of interest are transcribed in specific cell types. Therefore, neuronal proportion computed by using genome-wide DNA methylation data, and mRNA levels of neuronal marker RBFOX3, which correlates with neuronal proportion, were included as confounding factors in the analysis (for details, see ref. ${ }^{39}$ and Table 1 ).

\section{PDYN and OPRK1 gene expression in human dIPFC: absolute levels and cell-type specificity}

Receptor activation depends on concentration of its ligands, which may be produced in shortage or in excess relative to the receptor ${ }^{53}$. We examined whether a limiting factor in the DYN/KOR signaling in dIPFC may be defined at the level of PDYN or KOR (OPRK1) gene transcription by comparing absolute levels of $P D Y N$ and OPRK 1 mRNAs by ddPCR using cDNA prepared from total dIPFC tissue samples from control subjects (Fig. 1a). The $P D Y N$ mRNA levels were significantly 2 -fold lower compared to those of OPRK1 levels $\left(t_{(18)}=-5.3864, P=\right.$ $4.1 \times 10^{-5}$; Fig. 1a). Considering rapid DYN degradation and relatively large extracellular volume in which the peptides are diluted, the concentration of DYNs in the vicinity of the receptor molecules should be low resulting in the receptors' underactivation. Thus, transcriptional processes may already establish proportion in the expression of PDYN and KOR (OPRK1) genes rendering the DYN production to be a limiting factor in the DYN/ KOR signaling in dlPFC.

Expression levels of PDYN and KOR (OPRK1) in dlPFC may depend on proportion of cell type(s), specifically neurons, transcribing these genes in the tissue. To 

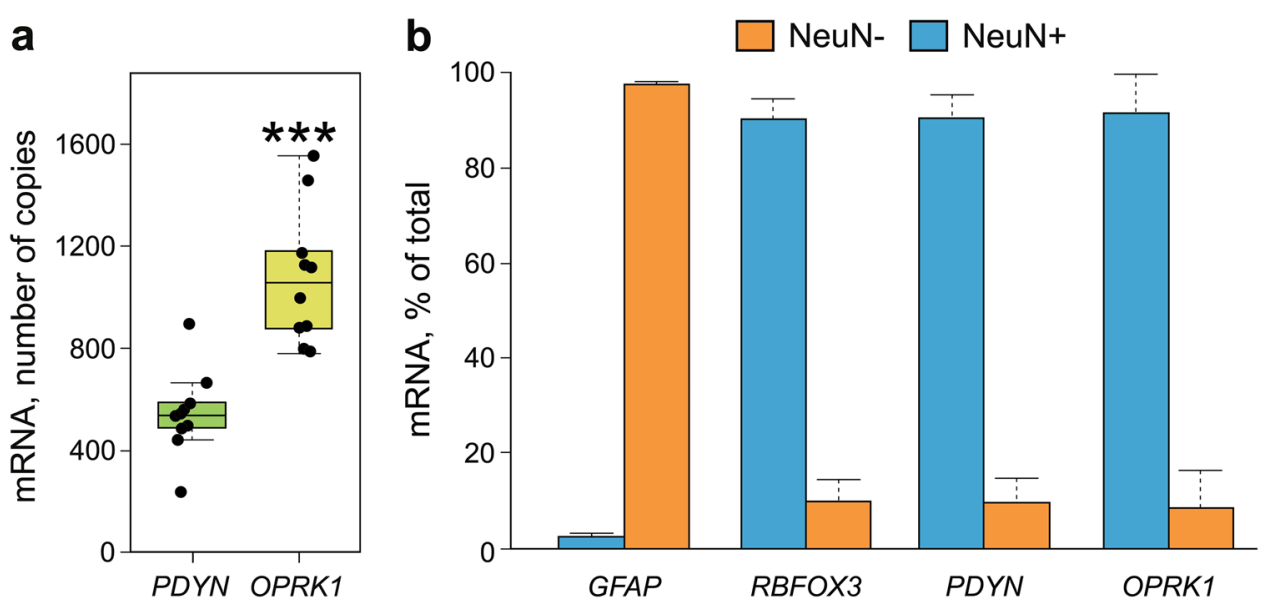

Fig. 1 Expression of the PDYN and KOR (OPRK1) genes in human dIPFC. a Absolute levels of PDYN and OPRK1 mRNAs measured by ddPCR using total tissue RNA ( $n=10$ control subjects), presented as a number of mRNA copies/ng of total RNA. Student's $t$-test, ${ }^{* * *} P<0.001$. In box plots, middle line is the median, box spans the interquartile range (IQR), and whiskers extend $1.5 \times I Q R$ from box limits. $\mathbf{b}$ Neuronal expression of PDYN and OPRK 1 genes in dIPFC. Levels of RBFOX3 (neuronal marker), PDYN, and OPRK1 mRNAs are high in neuronal nuclei (NeuN+), while those of GFAP (astrocyte marker) in non-neuronal (NeuN-) nuclei. Bar graphs show average mRNA amount \pm S.E.M. in NeuN+ and NeuN- fractions as \% of total amount of these mRNAs. Nuclei were isolated by FANS individually from dIPFC tissue of $n=3$ (used for analysis of PDYN, RBFOX3, and GFAP mRNAs) or $n=2$ (used for analysis of OPRK1 mRNA) subjects. mRNA levels were analyzed by ddPCR and normalized to total RNA content

confirm neuronal expression of PDYN and OPRK1 in human dlPFC, we analyzed levels of PDYN and KOR (OPRK1) mRNAs by ddPCR in neuronal and nonneuronal cell nuclei isolated from this tissue samples by FANS with antibodies against NeuN, the neuronal marker transcribed from the RBFOX3 gene (Fig. 1b). mRNAs of RBFOX3 and glial fibrillary acidic protein (GFAP) genes, the neuronal and astrocyte markers analyzed as positive and negative controls were localized in neuronal and nonneuronal nuclei, respectively. Both PDYN and OPRK1 mRNAs were highly enriched, approx. 10-fold in neuronal compared to non-neuronal nuclei (Fig. 1b). Neuronal proportion and mRNA levels of neuronal marker NeuN (RBFOX3) were then included as covariates in statistical models for comparison of PDYN and OPRK1 tissue expression levels between pathological and control brain.

\section{Effects of alcoholism on PDYN expression; influence of cell composition}

We next examined whether the tissue PDYN expression levels calculated as the ratio to reference genes were affected by alcoholism. PDYN mRNA levels in alcoholics compared to controls were found to be significantly lower, 1.24-fold (main effect of alcoholism, mean and 95\% CI estimated by bootstrap resampling, $-0.133[-0.220$, $-0.041], P=0.004$; Fig. 2a). When adjusted for the levels of RBFOX3 mRNA, a neuronal marker, or for cell composition, $P D Y N$ mRNA levels in alcoholics compared with controls were lower, respectively, with a trend $(-0.088$ [-0.183, 0.019], $P=0.086 ; 1.15$-fold; Fig. $2 \mathrm{~b})$, or significantly $(-0.166[-0.285,-0.051], P=0.006 ; 1.29$-fold;
Fig. 2c). Inclusion of smoking status as covariate in statistical models did not affect significance of differences identified in dIPFC (for tissue levels: $-0.122[-0.217$, $-0.012], P=0.019)$ and when corrected for cell composition $(-0.146[-0.265,-0.025], P=0.019)$. No effects of severity of alcohol use disorder $(P=0.834)$, mean lifetime alcohol consumption $(P=0.127)$, or lifetime alcohol consumption $(P=0.581)$ on $P D Y N$ expression were found. $P D Y N$ mRNA significantly correlated with RBFOX3 mRNA levels (main effect of RBFOX3 mRNA, mean and 95\% CI, 0.197 [0.068, 0.340], $P=0.007$; Fig. 2d), but not with neuronal proportion (Fig. 2e). These results suggest that $P D Y N$ transcription is downregulated in dIPFC in alcoholics. This decrease does not depend on changes in cell composition, and consistently PDYN does not correlate with neuronal proportion. However, PDYN expression may be associated with that of neuronal marker NeuN (RBFOX3).

\section{Effects of alcoholism on KOR expression; influence of cell composition}

No significant effects of alcoholism on KOR (OPRK1) mRNA were evident for the tissue levels (Fig. 3a) and also the levels adjusted for RBFOX3 expression levels (Fig. 3b) and for cell composition (Fig. 3c). Inclusion of smoking status as covariate revealed no differences between the subject groups. No effects of severity of alcohol use disorder $(P=0.768)$, mean lifetime alcohol consumption $(P$ $=0.415)$, or lifetime alcohol consumption $(P=0.479)$ on OPRK1 expression were found. No significant correlation was found between OPRK1 and RBFOX3 mRNA levels 
Tissue levels

a

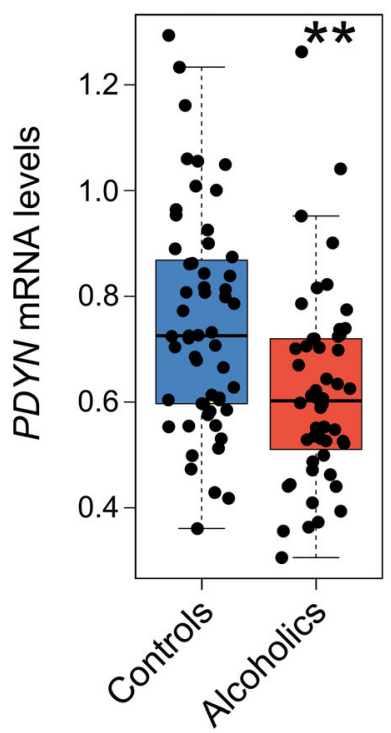

b

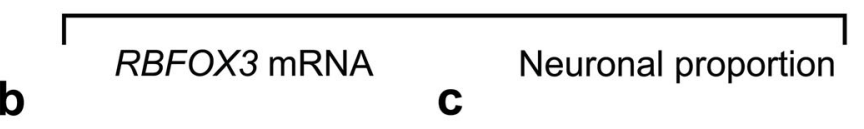

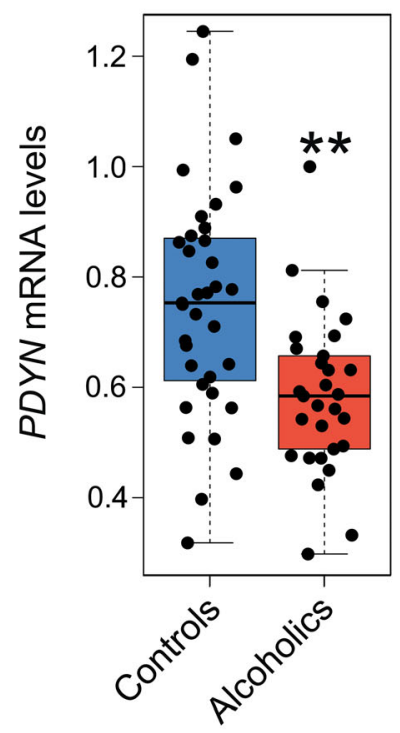

d

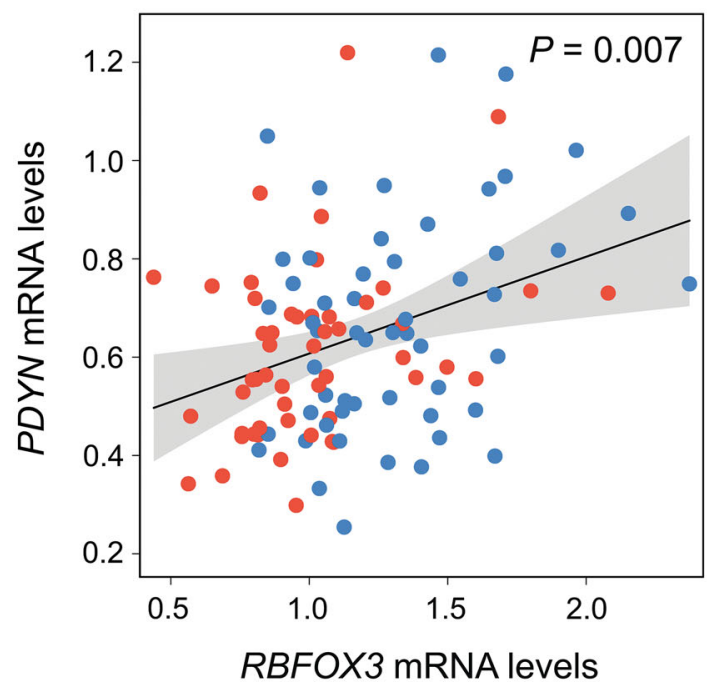

e
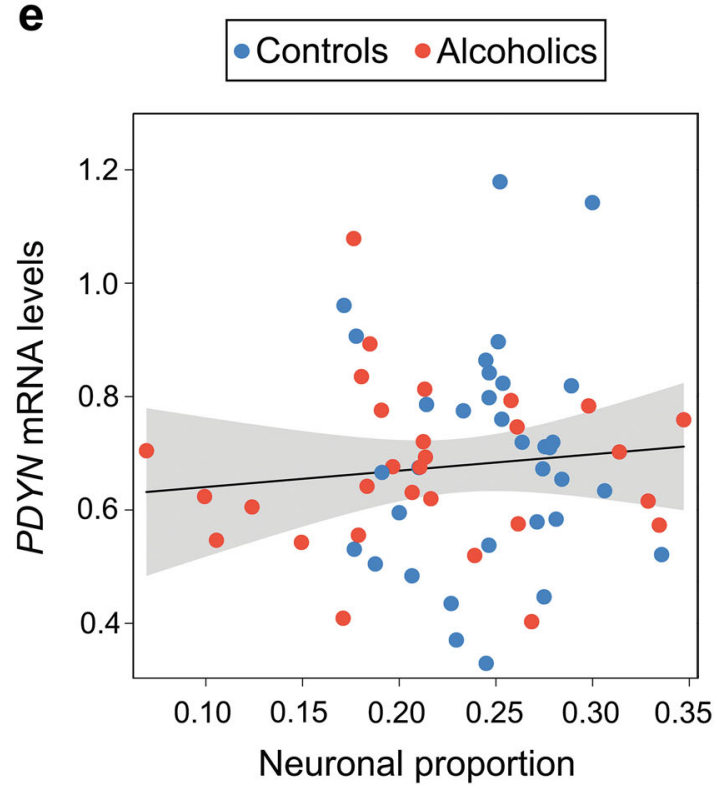

Fig. 2 Effects of alcoholism on the tissue PDYN expression levels, and the expression levels controlled for cell composition in human dIPFC. a PDYN mRNA levels were significantly lower in alcoholics $(P=0.004)$. $\mathbf{b}$ Expression of PDYN controlled for RBFOX3 mRNA levels differed between the two groups with a trend $(P=0.086)$. $\mathbf{c}$ Expression of PDYN controlled for neuronal proportion was significantly lower in alcoholics $(P=$ 0.006). d Relationship between PDYN and RBFOX3 mRNA levels; correlation was positive and significant $(P=0.007)$. e $P D Y N$ and neuronal proportion did not significantly correlate $(P=0.453)$. Data from the cohort of 55 controls and 53 alcoholics $(\mathbf{a}, \mathbf{b}, \mathbf{d})$, or the cohort of 35 controls and 30 alcoholics (c, e) were analyzed via linear regression. mRNA levels are shown in arbitrary units. In box plots middle line is the median, box spans the interquartile range (IQR), and whiskers extend 1.5 $\times 1 \mathrm{IQR}$ from box limits. Lines and shading represent the estimated slopes and $95 \%$ confidence intervals, respectively. ${ }^{* *} P<0.01$ by ordinary bootstrap with $5 \times 10^{5}$ nonparametric resampling of cases 
(Fig. 3d), and OPRK1 mRNA levels and neuronal proportion (Fig. 3e). These data imply that the number of neurons producing $P D Y N$ or KOR is not proportional to the total number of neurons in $\mathrm{dIPFC}$, and that the PDYN and KOR expressing neuronal subtypes and total neuronal population may be differentially affected by alcoholism.

\section{Correlation between PDYN and KOR}

The PDYN and OPRK1 genes may be co-expressed (i.e., transcriptionally co-regulated) and their co-expression pattern may be affected upon transition from normal to alcoholic state. To address this hypothesis we compared the slopes of regression lines for PDYN and OPRK1 mRNA levels between controls and alcoholics. PDYN and OPRK1 mRNAs correlated with a trend (main effect of OPRK1 mRNA, 0.309 [-0.003, 0.639], $P=0.061$; Fig. 4a), while interaction effect of alcoholism on PDYN-KOR correlation was not significant. Thus, correlation between $P D Y N$ and OPRK1 expression did not differ between alcoholics and controls; the slope of regression line was similar in alcoholics and controls. The effect of alcoholism was not sensitive to (i) changes in cell composition assessed using neuronal marker NeuN (RBFOX3) or neuronal proportion, and to (ii) smoking status. This suggests a low level of coordination in regulation of these two genes both in controls and alcoholics.

\section{Correlation between PDYN mRNA and dynorphin B, a mature peptide $P D Y N$ product}

$P D Y N$ mRNA correlated with dynorphin B, the peptide product of this gene (main effect of PDYN mRNA, 0.960 $[0.550,1.400], P=0.0001$; Fig. $4 \mathrm{~b}$ ), while no differences in the level of this endogenous kappa-agonist were found between alcoholics and controls (main effect of alcoholism, $0.052[-0.128,0.280], P=0.605)$.

\section{Effects of alcoholism-associated SNPs on PDYN and OPRK1 expression in controls and alcoholics}

The level of alcohol-dependent activation of endogenous opioid transmission might be genetically deter$\operatorname{mined}^{54,55}$. We therefore examined whether adaptive $P D Y N$ responses to alcohol may be modulated by PDYN SNPs associated with alcoholism including promoter SNP rs1997794, and SNPs rs6045819 and rs2235749 located in coding exon 4 and $3^{\prime}$ untranslated region ( $3^{\prime}$-UTR) of the gene $^{56,57}$. PDYN promoter SNP (rs1997794) was also found to be associated with better episodic memory scores in elderly humans ${ }^{22}$, and may form non-canonical AP-1 binding site and influence gene expression in human brain $^{58}$.

The Fisher's exact test revealed no significant difference in distribution of $P D Y N$ promoter SNP rs1997794 genotypes $(P=0.329)$ between alcoholics and control subjects
(CC and CT genotypes vs. TT genotype; subjects with the $\mathrm{C}$, high-risk genotype were pooled); or other SNPs (rs6045819, $P=0.528 ;$ rs2235749, $P=0.679$; rs6985606, $P=0.326)$.

Two-way ANCOVA with group (controls vs. alcoholics) and PDYN promoter SNP rs1997794 genotype as between factors revealed a significant effect of group $\times$ genotype interaction $(-0.179[-0.320,-0.030], P=0.016)$. Main effect of genotype was significant $(0.186$ [0.089, 0.287], $P$ $=0.0003)$, while main effect of alcoholism was not significant $(-0.022[-0.119,0.077], P=0.659)$. For the combined CC and CT genotypes post hoc $t$-test showed downregulation of PDYN (1.37-fold; $P=0.0002)$ in alcoholics (Fig. 4c). The effect of alcoholism $\times$ genotype interaction was not sensitive to changes in cell composition assessed using neuronal marker NeuN (RBFOX3) $(-0.148[-0.289,-0.0002], P=0.044)$. No other SNPs tested significantly affected $P D Y N$ or OPRK1 expression between controls and alcoholics.

\section{Discussion}

The main finding of our study is downregulation of PDYN expression in dIPFC of alcoholics. DYNs have a role in learning and memory acquisition, while their elevated levels are deteriorating for cognitive processes ${ }^{17-19}$. Impairments of spatial learning and memory by ethanol exposure were found to be mediated through activation of the $\mathrm{KOR}^{16}$. KOR antagonists abrogated alcohol-induced pathological processes. In this framework, the decrease in $P D Y N$ expression in dIPFC may be interpreted as adaptation that may counteract cognitive decline developed over the years of heavy alcohol drinking and withdrawal.

Postmortem studies of PDYN and KOR (OPRK1) regulation in addicted human brain are limited in a number and mostly focused on the striatal subregions. PDYN was found to be downregulated in NAc core in heroin addicts $^{59}$, while no changes in PDYN or DYN and KOR protein expression in NAc of cocaine addicts ${ }^{60,61}$ were revealed. The PDYN and KOR (OPRK1) gene expression and DYN levels were reported to be elevated in dorsal striatum of cocaine addicts ${ }^{60,61}$, while the PDYN mRNA levels were decreased in dorsal striatum in alcoholics ${ }^{44}$ and in caudate nucleus of cocaine addicts carrying PDYN SNP variant associated with cocaine/alcohol codependence $^{57}$. The absence of consistency in these data is likely due to underpowered postmortem human brain analysis in some of these works. Sample size calculation for PDYN expression levels in alcoholics and controls demonstrated that analyses with $n \leq 30$, including our pilot study with 14 alcoholics and 14 controls, which were not overlapping with the current sample, and which suggested modest elevation in PDYN expression in dlPFC of alcoholics ${ }^{35}$, are likely underpowered. "Medium" size effects ${ }^{62}$ may be detected with $n=90$ or larger sample size. In this study, 


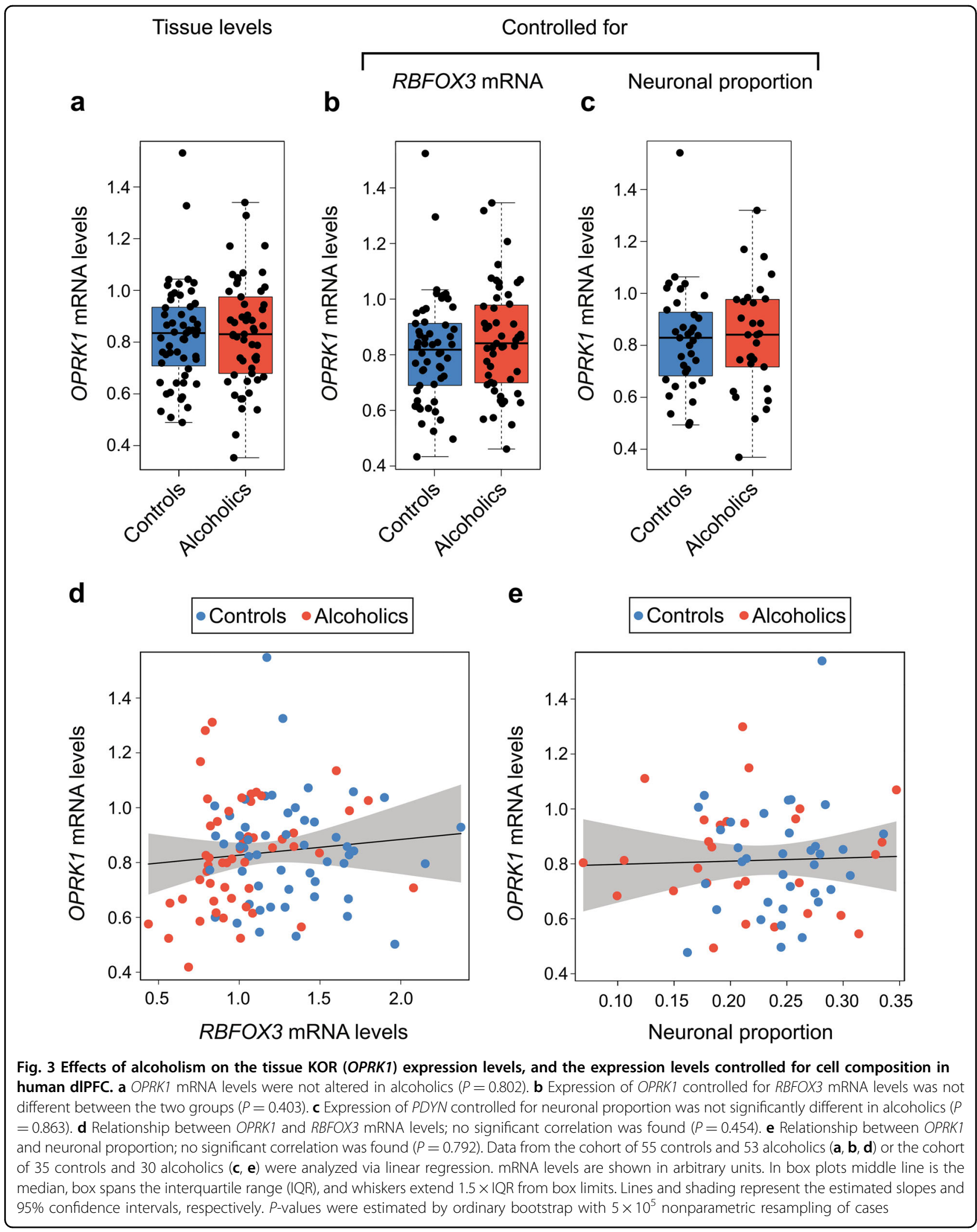



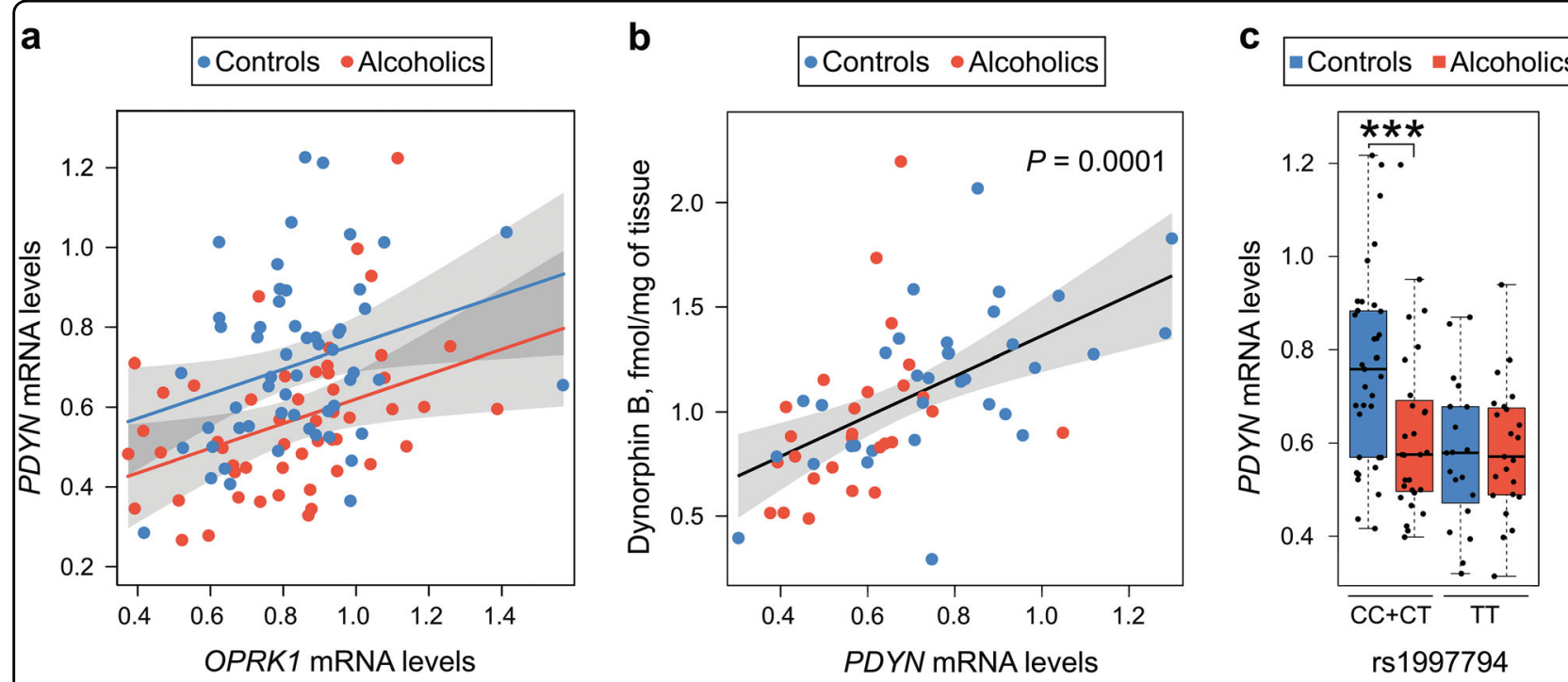

Fig. 4 Relationship between PDYN and KOR (OPRK1) mRNAs (a), and between PDYN mRNA and dynorphin B (b) in dIPFC of controls and alcoholics. Effects of PDYN promoter SNP rs1997794 genotype on the PDYN tissue expression levels in dIPFC of alcoholic and control subjects (c). a Linear regression model corrected for age, PMl, brain $\mathrm{pH}$, and RQI revealed a trend for positive correlation between PDYN and OPRK1 mRNAs in the combined controls and alcoholics group $(n=108$; main effect of OPRK1 mRNA; $P=0.061)$. Effects of alcoholism $\times$ OPRK1 mRNA interaction on PDYN mRNA levels were not significant ( $n=55$ controls and 53 alcoholics; $P=0.998$ ), demonstrating no difference in the slope of the regression lines between the two subject groups. $\mathbf{b}$ Linear regression model corrected for age, $\mathrm{PMl}$, brain $\mathrm{pH}$, and RQI revealed a positive correlation between PDYN mRNA and dynorphin $B$ in the combined controls and alcoholics group $(n=68$; main effect of PDYN mRNA; $P=0.0001)$. Effect of alcoholism on dynorphin B levels was not significant $(P=0.605)$. c Significant effect of alcoholism $\times$ genotype interaction on PDYN mRNA levels was found $(n=55$ controls and 53 alcoholics; $P=0.016$ ). PDYN mRNA levels were significantly 1.37-fold lower in alcoholics carrying $C$ (combined CC and CT genotypes, $n=28$ ) high-risk allele of rs 1997794 ( $P=0.0001)$ compared to control subjects with the same genotype $(n=35)$. mRNA levels are shown in arbitrary units, dynorphin B levels as fmol/mg of tissue. In box plots middle line is the median, box spans the interquartile range (IQR), and whiskers extend $1.5 \times I Q R$ from box limits. Lines and shading represent the estimated slopes and $95 \%$ confidence intervals, respectively. P-values were estimated by ordinary bootstrap with $5 \times 10^{5}$ resampling of cases. ${ }^{* *} P<0.001$ by post hoc $t$-test

we aimed to detect "large" effects by ensuring final sample size $n \geq 90$. Totally 108 subjects were analyzed that is likely the largest cohort of alcoholics and controls investigated to date. In addition, we applied a more stringent statistical analysis that included available confounding factors (age, PMI, brain $\mathrm{pH}, \mathrm{RQI}$, and smoking status). Alcoholism is associated with the decline in neuronal proportion in $\mathrm{dlPFC}^{37-39}$. Inclusion of changes in cell composition did not affect the significance of differences between the groups in PDYN expression, thus demonstrating that this is a robust phenomenon. Based on these issues we may conclude that the identified differences are characteristic of the analyzed population of alcoholics, who were males of European descent. Importantly, the in vitro study supports this notion by demonstrating a significant decrease in the expression of $P D Y N$ in human neuroblastoma $\mathrm{SH}-\mathrm{SY} 5 \mathrm{Y}$ cells following exposure to ethanol $^{63}$.

This study focuses on changes in expression and coexpression levels of the PDYN and KOR genes in alcoholics. Statistical analysis did not reveal effects of cell composition, suggesting that the observed expression differences may be caused primarily by adaptations in transcriptional mechanisms. These transcriptional changes may have functional consequences contributing to the downregulation of the DYN/KOR circuits in dIPFC of addicted individuals. In dIPFC the absolute expression levels of $P D Y N$ were significantly lower compared to those of KOR (OPRK1). Thus, PDYN expression may be a limiting factor in the DYN/KOR signaling, while its downregulation suggests the diminished efficacy of the DYN/KOR signaling in dlPFC of human alcoholics.

Development of drug/alcohol dependence may be viewed as a maladaptive habit formation during transition from recreational to compulsive use that is associated with a diminishing cognitive control over drug seeking and taking behavior ${ }^{27,29,64-66}$. These processes are characterized by a shift from prefrontal cortical to striatal control over drug/alcohol use, and a progression from the ventral to dorsal striatum in the addicted brain ${ }^{64,67}$. This shift is associated with the downregulation of the DYN/ KOR system in both dIPFC (present study), and caudate and putamen ${ }^{44}$. Whether the paralleled DYN/KOR adaptations in dIPFC and dorsal striatum in alcoholics is a part of molecular processes underlying transition to the addictive state is a subject for future studies. 
Associations between alcohol dependence and nine SNPs in the PDYN promoter and 3'-UTR and five SNPs in intron 2 of OPRK1 were previously reported ${ }^{56}$. We selected three PDYN SNPs (rs1997794 located in the promoter, rs6045819 in coding exon 4, and rs2235749 in 3 '-UTR) and one OPRK1 SNP (rs6985606 located in intron 2) with highest significance of association (with $P<$ 0.01) for analysis. PDYN promoter SNP rs1997794 and also 3'-UTR SNP rs910080 (located in the haplotype block with rs2235749) were also found to be associated with better episodic memory scores in elderly humans ${ }^{22}$. PDYN promoter SNP rs1997794 associated with alcoholism $^{56,57}$ may form non-canonical AP-1 binding site and influence gene expression in human brain ${ }^{58,68}$.

For $P D Y N$ promoter SNP rs1997794, a significant effect of group $\times$ genotype interaction and significant main effect of genotype were found. PDYN downregulation was significant in the subgroup of subjects carrying $C$, highrisk allele of $P D Y N$ SNP rs1997794. This finding corroborates previous findings for the putamen, where PDYN mRNA and Leu-enkephalin-Arg, a peptide derived from PDYN, were significantly downregulated in the same subgroup of subjects ${ }^{44}$. No other SNPs tested significantly affected PDYN or OPRK1 expression between controls and alcoholics.

As limitations, the identified associations may be only applicable to males of European descent because no female subjects and no other ethnic groups were analyzed. Human subjects were paired between two groups according to age, PMI, and brain $\mathrm{pH}$ and all confounding factors (age, PMI, brain $\mathrm{pH}, \mathrm{RQI}$, smoking status) were included in the analysis (Supplementary Table 1). The postmortem human findings may be interpreted in two ways, either as molecular adaptations in DYN/KOR expression patterns caused by heavy alcohol consumption and withdrawal or as manifestation of inherited molecular differences between controls and alcoholics.

The important limitation of the study is the absence of experimental background for functional implementation of the molecular findings at both molecular/cellular and behavioral levels. While PDYN mRNA correlated well with dynorphin $B$, the peptide product of this gene, no differences in the level of this endogenous kappa-agonist were found between alcoholics and controls. The DYN levels are regulated though synthesis and trafficking of their protein precursor molecule, processing of this molecule to mature opioid peptides, and release and degradation of the peptides. Furthermore there is a mismatch in anatomical localization of mRNA and mature peptide products. This mismatch occurs due to trafficking of the protein precursor molecules along neuronal projections to axon terminals in other brain areas, where these molecules are processed to mature DYNs ${ }^{47}$. These processes may be affected in alcoholic brain and, while not destroying the correlation, may shadow less pronounced differences between alcoholics and controls. We did not analyze relationship between the adaptations in the DYN/KOR system and cognate status of the individuals under study due to the absence of available premortal data. The functional consequence of PDYN downregulation in human alcoholics could be explored by analyzing the binding potential of selective kappa-opioid ligands, the development of which is rapidly progressing ${ }^{69}$.

In summary, our findings support the notion that the DYN/KOR signaling is dysregulated in human alcoholics. Chronic effects of various addictive substances may cause similar area-nonspecific transcriptional adaptations-the common downstream molecular syndrome mediating the lasting nature of the addictive state ${ }^{66}$. Downregulation of DYNs in dIPFC (present study), and dorsal striatum of alcoholics $^{44}$ and cocaine addicts ${ }^{57}$ may be a part of this general adaptive mechanism.

\section{Acknowledgements}

We are grateful to Prof. Ingrid Nylander for providing antibodies against opioid peptides. This work was supported by grants from the Swedish Science Research Council (K2014-62X-12190-19-5), and Swedish Council for Working Life and Social Research (2009-1709, 259-2012-23) to G.B. NSW BTRC was supported by the US Government under the National Institutes of Health, USA (NIAAA012725-15) and the University of Sydney.

\section{Author details}

1Division of Biological Research on Drug Dependence, Department of Pharmaceutical Biosciences, Uppsala University, SE-751 24 Uppsala, Sweden. ${ }^{2}$ Department of Psychiatry and Psychology, Mayo Clinic College of Medicine, Rochester, MN 55905, USA

\section{Conflict of interest}

The authors declare that they have no conflict of interest.

\section{Publisher's note}

Springer Nature remains neutral with regard to jurisdictional claims in published maps and institutional affiliations.

The online version of this article (https://doi.org/10.1038/s41398-017-0075-5) contains supplementary material.

Received: 8 August 2017 Revised: 2 October 2017 Accepted: 1 November 2017

Published online: 20 June 2018

\section{References}

1. Loeber, S., Duka, T., Welzel, H., Nakovics, H., Heinz, A. \& Flor, H. et al. Impairment of cognitive abilities and decision making after chronic use of alcohol: the impact of multiple detoxifications. Alcohol Alcohol. 44, 372-381 (2009).

2. Park, S. Q., Kahnt, T., Beck, A., Cohen, M. X., Dolan, R. J. \& Wrase, J. et al. Prefrontal cortex fails to learn from reward prediction errors in alcohol dependence. J. Neurosci. 30, 7749-7753 (2010).

3. Leber, W. R., Jenkins, R. L. \& Parsons, O. A. Recovery of visual-spatial learning and memory in chronic alcoholics. J. Clin. Psychol. 37, 192-197 (1981).

4. Oscar-Berman, M. \& Marinkovic, K. Alcohol: effects on neurobehavioral functions and the brain. Neuropsychol. Rev. 17, 239-257 (2007).

5. Oslin, D. W. \& Cary, M. S. Alcohol-related dementia: validation of diagnostic criteria. Am. J. Geriatr. Psychiatry 11, 441-447 (2003). 
6. Le Berre, A. P., Fama, R. \& Sullivan, E. V. Executive functions, memory, and social cognitive deficits and recovery in chronic alcoholism: a critical review to inform future research. Alcohol. Clin. Exp. Res. 41, 1432-1443 (2017).

7. Mukamal, K. J., Kuller, L. H., Fitzpatrick, A. L., Longstreth, W. T. Jr., Mittleman, M. A. \& Siscovick, D. S. Prospective study of alcohol consumption and risk of dementia in older adults. J. Am. Med. Assoc. 289, 1405-1413 (2003).

8. Saunders, P. A., Copeland, J. R. M., Dewey, M. E., Davidson, I. A., Mcwilliam, C. \& Sharma, V. et al. Heavy drinking as a risk factor for depression and dementia in elderly men-findings from the liverpool longitudinal community study. Br. J. Psychiatry 159, 213-216 (1991).

9. Jarvenpaa, T., Rinne, J. O., Koskenvuo, M., Raiha, I. \& Kaprio, J. Binge drinking in midlife and dementia risk. Epidemiology 16, 766-771 (2005).

10. Crews, F. T. \& Boettiger, C. A. Impulsivity, frontal lobes and risk for addiction Pharmacol. Biochem. Behav. 93, 237-247 (2009).

11. Robbins, T. W., Gillan, C. M., Smith, D. G., de Wit, S. \& Ersche, K. D. Neurocognitive endophenotypes of impulsivity and compulsivity: towards dimensional psychiatry. Trends Cogn. Sci. 16, 81-91 (2012).

12. Abraham, A. D., Fontaine, H. M., Song, A. J., Andrews, M. M., Baird, M. A. \& Kieffer, B. L. et al. Kappa opioid receptor activation in dopamine neurons disrupts behavioral inhibition. Neuropsychopharmacology 43, 362-372 (2017)

13. Negrete, R., Garcia Gutierrez, M. S., Manzanares, J. \& Maldonado, R. Involvement of the dynorphin/KOR system on the nociceptive, emotional and cognitive manifestations of joint pain in mice. Neuropharmacology 116, 315-327 (2017).

14. Fadda, F. \& Rossetti, Z. L. Chronic ethanol consumption: from neuroadaptation to neurodegeneration. Prog. Neurobiol. 56, 385-431 (1998).

15. Stephens, D. N. \& Duka, T. Review. Cognitive and emotional consequences of binge drinking: role of amygdala and prefrontal cortex. Philos. Trans. R. Soc. Lond. B Biol. Sci. 363, 3169-3179 (2008).

16. Kuzmin, A., Chefer, V., Bazov, I., Meis, J., Ogren, S. O. \& Shippenberg, T. et al. Upregulated dynorphin opioid peptides mediate alcohol-induced learning and memory impairment. Transl. Psychiatry 3, e310 (2013).

17. Sandin, J., Nylander, I., Georgieva, J., Schott, P. A., Ogren, S. O. \& Terenius, L. Hippocampal dynorphin B injections impair spatial learning in rats: a kappaopioid receptor-mediated effect. Neuroscience 85, 375-382 (1998).

18. Jiang, H. K., Owyang, V. V., Hong, J. S. \& Gallagher, M. Elevated dynorphin in the hippocampal formation of aged rats: relation to cognitive impairment on a spatial learning task. Proc. Natl Acad. Sci. USA 86, 2948-2951 (1989).

19. Nguyen, X. V., Masse, J., Kumar, A., Vijitruth, R., Kulik, C. \& Liu, M. et al. Prodynorphin knockout mice demonstrate diminished age-associated impairment in spatial water maze performance. Behav. Brain Res. 161, 254-262 (2005).

20. Carey, A. N., Lyons, A. M., Shay, C. F., Dunton, O. \& McLaughlin, J. P. Endogenous kappa opioid activation mediates stress-induced deficits in learning and memory. J. Neurosci. 29, 4293-4300 (2009).

21. Yakovleva, T., Marinova, Z., Kuzmin, A., Seidah, N. G., Haroutunian, V. \& Terenius, L. et al. Dysregulation of dynorphins in Alzheimer disease. Neurobiol. Aging $\mathbf{2 8}$, 1700-1708 (2007).

22. Kolsch, H., Wagner, M., Bilkei-Gorzo, A., Toliat, M. R., Pentzek, M. \& Fuchs, A. et al. Gene polymorphisms in prodynorphin (PDYN) are associated with episodic memory in the elderly. J. Neural Transm. 116, 897-903 (2009).

23. Kuzmin, A., Liljequist, S., Meis, J., Chefer, V., Shippenberg, T. \& Bakalkin, G. Repeated moderate-dose ethanol bouts impair cognitive function in Wistar rats. Addict. Biol. 17, 132-140 (2012).

24. Chefer, V., Meis, J., Wang, G., Kuzmin, A., Bakalkin, G. \& Shippenberg, T. Repeated exposure to moderate doses of ethanol augments hippocampal glutamate neurotransmission by increasing release. Addict. Biol. 16, 229-237 (2011).

25. Koob, G. F. The dark side of emotion: the addiction perspective. Eur. J. Pharmacol. 753, 73-87 (2015).

26. Butelman, E. R., Yuferov, V. \& Kreek, M. J. Kappa-opioid receptor/dynorphin system: genetic and pharmacotherapeutic implications for addiction. Trends Neurosci. 35, 587-596 (2012).

27. Shippenberg, T. S., Zapata, A. \& Chefer, V. I. Dynorphin and the pathophysiology of drug addiction. Pharmacol. Ther. 116, 306-321 (2007).

28. Walker, B. M. \& Koob, G. F. Pharmacological evidence for a motivational role of kappa-opioid systems in ethanol dependence. Neuropsychopharmacology 33, 643-652 (2008).

29. Wee, S. \& Koob, G. F. The role of the dynorphin-kappa opioid system in the reinforcing effects of drugs of abuse. Psychopharmacology 210, 121-135 (2010).

30. Kreek, M. J., Levran, O., Reed, B., Schlussman, S. D., Zhou, Y. \& Butelman, E. R. Opiate addiction and cocaine addiction: underlying molecular neurobiology and genetics. J. Clin. Invest. 122, 3387-3393 (2012).
31. Schlosburg, J. E., Whitfield, T. W. Jr., Park, P. E., Crawford, E. F., George, O. \& Vendruscolo, L. F. et al. Long-term antagonism of kappa opioid receptors prevents escalation of and increased motivation for heroin intake. J. Neurosci. 33, 19384-19392 (2013).

32. Nealey, K. A., Smith, A. W., Davis, S. M., Smith, D. G. \& Walker, B. M. Kappa-opioid receptors are implicated in the increased potency of intra-accumbens nalmefene in ethanol-dependent rats. Neuropharmacology 61, 35-42 (2011).

33. Walker, B. M., Zorrilla, E. P. \& Koob, G. F. Systemic kappa-opioid receptor antagonism by nor-binaltorphimine reduces dependence-induced excessive alcohol self-administration in rats. Addict. Biol. 16, 116-119 (2011).

34. Kissler, J. L., Sirohi, S., Reis, D. J., Jansen, H. T., Quock, R. M. \& Smith, D. G. et al. The one-two punch of alcoholism: role of central amygdala dynorphins/ kappa-opioid receptors. Biol. Psychiatry 75, 774-782 (2014).

35. Bazov, I., Kononenko, O., Watanabe, H., Kuntic, V., Sarkisyan, D. \& Taqi, M. M. et al. The endogenous opioid system in human alcoholics: molecular adaptations in brain areas involved in cognitive control of addiction. Addict. Biol. 18, 161-169 (2013).

36. Bazov, l., Sarkisyan, D., Kononenko, O., Watanabe, H., Yakovleva, T., \& Hansson, A.C. et al. Dynorphin and k-Opioid Receptor Dysregulation in the Dopaminergic Reward System of Human Alcoholics. Mol Neurobiol. 2018 Jan 30. http://doi.org/10.1007/s12035-017-0844-4.

37. de la Monte, S. M. \& Kril, J. J. Human alcohol-related neuropathology. Acta Neuropathol. 127, 71-90 (2014).

38. Zahr, N. M., Kaufman, K. L. \& Harper, C. G. Clinical and pathological features of alcohol-related brain damage. Nat. Rev. Neurol. 7, 284-294 (2011).

39. Sarkisyan, D., Bazov, I., Watanabe, H., Kononenko, O., Syvanen, A. C. \& Schumann, G. et al. Damaged reward areas in human alcoholics: neuronal proportion decline and astrocyte activation. Acta Neuropathol. 133, 485-487 (2017).

40. Harper, C., Kril, J. \& Daly, J. Does a "moderate" alcohol intake damage the brain? J. Neurol. Neurosurg. Psychiatry 51, 909-913 (1988).

41. Sheedy, D., Garrick, T., Dedova, I., Hunt, C., Miller, R. \& Sundqvist, N. et al. An Australian Brain Bank: a critical investment with a high return! Cell Tissue Bank. 9, 205-216 (2008).

42. Vandesompele, J., De Preter, K., Pattyn, F., Poppe, B., Van Roy, N., De Paepe, A. et al. Accurate normalization of real-time quantitative RT-PCR data by geometric averaging of multiple internal control genes. Genome Biol. 3, RESEARCH0034 (2002).

43. Johansson, S., Fuchs, A., Okvist, A., Karimi, M., Harper, C. \& Garrick, T. et al. Validation of endogenous controls for quantitative gene expression analysis: application on brain cortices of human chronic alcoholics. Brain Res. 1132, 20-28 (2007).

44. Sarkisyan, D., Hussain, M. Z., Watanabe, H., Kononenko, O., Bazov, I. \& Zhou, X. et al. Downregulation of the endogenous opioid peptides in the dorsal striatum of human alcoholics. Front. Cell Neurosci. 9, 187 (2015).

45. Christensson-Nylander, I., Nyberg, F., Ragnarsson, U. \& Terenius, L. A general procedure for analysis of proenkephalin B derived opioid peptides. Regul. Pept. 11, 65-76 (1985).

46. Merg, F., Filliol, D., Usynin, I., Bazov, I., Bark, N. \& Hurd, Y. L. et al. Big dynorphin as a putative endogenous ligand for the kappa-opioid receptor. J. Neurochem. 97, 292-301 (2006).

47. Yakovleva, T., Bazov, I., Cebers, G., Marinova, Z., Hara, Y. SpringerAmpamp; Ahmed, A. et al. Prodynorphin storage and processing in axon terminals and dendrites. FASEB J. 20, 2124-2126 (2006).

48. McDermott, G. P., Do, D., Litterst, C. M., Maar, D., Hindson, C. M. \& Steenblock, E. $\mathrm{R}$. et al. Multiplexed target detection using DNA-binding dye chemistry in droplet digital PCR. Anal. Chem. 85, 11619-11627 (2013).

49. Hindson, C. M., Chevillet, J. R., Briggs, H. A., Gallichotte, E. N., Ruf, I. K. \& Hindson, B. J. et al. Absolute quantification by droplet digital PCR versus analog realtime PCR. Nat. Methods 10, 1003-1005 (2013).

50. Guintivano, J., Aryee, M. J. \& Kaminsky, Z. A. A cell epigenotype specific model for the correction of brain cellular heterogeneity bias and its application to age, brain region and major depression. Epigenetics 8, 290-302 (2013).

51. Faraway, J. J. Practical Regression and ANOVA Using R (University of Bath, Bath, UK, 2002).

52. Fox, J. \& Weisberg, S. Cox Proportional-Hazards Regression for Survival Data in $R$. An Appendix to an R Companion to Applied Regression (SAGE Publications, Thousand Oaks, 2011).

53. Kononenko, O., Galatenko, V., Andersson, M., Bazov, I., Watanabe, H., \& Zhou, X. W. et al. Intra- and interregional coregulation of opioid genes: broken symmetry in spinal circuits. FASEB J. 31, 1953-1963 (2017). 
54. Heilig, M., Goldman, D., Berrettini, W. \& O'Brien, C. P. Pharmacogenetic approaches to the treatment of alcohol addiction. Nat. Rev. Neurosci. 12, 670-684 (2011).

55. Levran, O., Yuferov, V. \& Kreek, M. J. The genetics of the opioid system and specific drug addictions. Hum. Genet. 131, 823-842 (2012).

56. Xuei, X., Dick, D., Flury-Wetherill, L., Tian, H. J., Agrawal, A. \& Bierut, L. et al. Association of the kappa-opioid system with alcohol dependence. Mol. Psychiatry 11, 1016-1024 (2006)

57. Yuferov, V., Ji, F., Nielsen, D. A., Levran, O., Ho, A. \& Morgello, S. et al. A functional haplotype implicated in vulnerability to develop cocaine dependence is associated with reduced PDYN expression in human brain. Neuropsychopharmacology 34, 1185-1197 (2009).

58. Taqi, M. M., Bazov, I., Watanabe, H., Nyberg, F., Yakovleva, T. \& Bakalkin, G Prodynorphin promoter SNP associated with alcohol dependence forms noncanonical AP-1 binding site that may influence gene expression in human brain. Brain Res. 1385, 18-25 (2011).

59. Drakenberg, K., Nikoshkov, A., Horvath, M. C., Fagergren, P., Gharibyan, A. \& Saarelainen, K. et al. Mu opioid receptor A118G polymorphism in association with striatal opioid neuropeptide gene expression in heroin abusers. Proc. Natl Acad. Sci. USA 103, 7883-7888 (2006)

60. Frankel, P. S., Alburges, M. E., Bush, L., Hanson, G. R. \& Kish, S. J. Striatal and ventral pallidum dynorphin concentrations are markedly increased in human chronic cocaine users. Neuropharmacology 55, 41-46 (2008).

61. Hurd, Y. L. \& Herkenham, M. Molecular alterations in the neostriatum of human cocaine addicts. Synapse 13, 357-369 (1993).
62. Cohen, J., Cohen, P., West, S. G., Aiken, L. S. Applied Multiple Regression/Correlation Analysis for the Behavioral Sciences (Routledge, Oxford, UK, 2013).

63. D'Addario, C., Ming, Y., Ogren, S. O. \& Terenius, L. The role of acetaldehyde in mediating effects of alcohol on expression of endogenous opioid system genes in a neuroblastoma cell line. FASEB J. 22, 662-670 (2008).

64. Everitt, B. J. \& Robbins, T. W. Neural systems of reinforcement for drug addiction: from actions to habits to compulsion. Nat. Neurosci. 8, 1481-1489 (2005).

65. Butelman, E. R., Yuferov, V. \& Kreek, M. J. Kappa-opioid receptor/dynorphin system: genetic and pharmacotherapeutic implications for addiction. Trends Neurosci. 35, 587-596 (2012)

66. Robison, A. J. \& Nestler, E. J. Transcriptional and epigenetic mechanisms of addiction. Nat. Rev. Neurosci. 12, 623-637 (2011).

67. Vollstadt-Klein, S., Wichert, S., Rabinstein, J., Buhler, M., Klein, O. \& Ende, G. et al. Initial, habitual and compulsive alcohol use is characterized by a shift of cue processing from ventral to dorsal striatum. Addiction 105, 1741-1749 (2010).

68. Babbitt, C. C., Silverman, J. S., Haygood, R., Reininga, J. M., Rockman, M. V. \& Wray, G. A. Multiple functional variants in cis modulate PDYN expression. Mol. Biol. Evol. 27, 465-479 (2010).

69. Li, S., Cai, Z., Zheng, M. Q., Holden, D., Naganawa, M., \& Lin, S. F. et al. A novel 18F-labeled kappa opioid receptor antagonist as PET radiotracer: synthesis and in vivo evaluation of 18F-LY2459989 in non-human primates. J. Nucl. Med. 59 140-146 (2017). 